\title{
Ataxia Telangiectasia: Case Report from a Rural Hospital in Nepal and Current Management Recommendation
}

\author{
George SM1, Mathews MC $^{2}$
}

\begin{abstract}
It is important though challenging to make accurate diagnosis of neurodegenerative conditions in children and initiate the right treatment. A 14 years old boy presented with deterioration in speech, mobility and performance of daily activities and frequent chest infections over four years. Aided ataxic gait, dysarthria, stooped posture, generalised hypotonia and weakness and ocular telangiectasia were noted. We present a case of Ataxia telangiectasia from a rural hospital in Nepal.
\end{abstract}

\section{Introduction}

$\mathrm{P}$ aediatric neuro-degenerative conditions can be challenging in resource poor settings- both for the patients with financial constraints, and the clinicians with limited facilities. Ataxia, not an unusual presentation in children, can pose diagnostic dilemmas. We report a patient with Ataxia Talangiectasia (AT), a rare progressive genetic neurological condition. AT, an autosomal recessive condition, presents in early childhood with ataxia, telangiectasia (dilated small blood vessels), immunodeficiency, frequent sino-pulmonary infections and increased susceptibility to malignancies ${ }^{1,2,3}$.

\section{The Case}

A 14 years old Nepali boy from a lower middle class Hindu Brahmin family presented with history of inability to walk independently, slurred speech, difficulty in writing, and lack of independence in performing day to day activities to the Paediatric outpatient clinic in this rural hospital. He was the first born of nonconsanguineous parents, and there were no similar conditions in the family. Born at term by normal vaginal delivery in hospital, there were no neonatal problems of note. His growth and neurodevelopment was normal until nine years.

At nine years, he developed difficulty in walking with frequent falls and ataxia. He also developed slurring of speech and difficulty in writing. A few months later he also developed drooling, difficulty in chewing and swallowing. He had blood shot eyes and 'unusual birth marks' behind his ears and on the back of his hands when these symptoms began. The birth marks were red in colour, looked like collection of small blood vessels, have disappeared in a few years. The abnormal ocular blood vessels (telangiectasia) persisted
'Dr. Susan Mary George, MBBS, MRCPCH, PhD, Visiting Paediatrician, ${ }^{2}$ Prof. MC Mathews, MBBS MD (Paed), PhD (Neuro), DNB (MCH), DCBR (Lon), DPH, DADR, DFDR, Professor and Consultant Developmental Neurologist, Department of Developmental Paediatrics and Child Neurology, MOSC Medical College, Kolenchery, India.

\section{Address for correspondence:}

Dr. Susan Mary George

E-mail: susanmarygeorge@hotmail.com

\section{How to cite}

George SM, Mathews MC. Ataxia Telangiectasia: Case Report from a Rural Hospital in Nepal and Current Management Recommendation. J Nepal Paediatr Soc 2014;34(2):138-140.

doi: http://dx.doi.org/10.3126/jnps.v34i2.9694

This work is licensed under a Creative Commons Attribution 3.0 License.

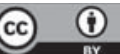

(Figure1). Medical opinion was sought at the time of onset of symptoms, provided no clear diagnosis.

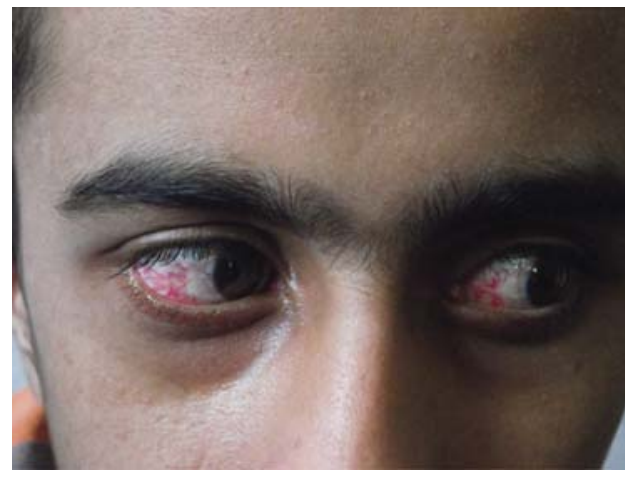

Fig 1: Telangiectasia seen in both eyes. 
The difficulty in walking worsened, and the patient became non-ambulatory at the age of 11 . He also developed a stooped posture and inability to sit without support over the last 3 years. Speech has gradually worsened, and he now has a soft, slow, scanning speech. Chewing and swallowing is difficult, and he is only able to take a soft, semi-solid diet now.

On examination he was thinly built, and appeared to have generalised wasting. There was mild drooling. He sat with a stooped posture. He had a slow unstable gait (aided by one person) with evidence of marked weakness of the lower limb muscles. Neurological examination revealed cerebellar signs- trunkal ataxia, nystagmus, oculo-motor dyspraxia, intentional tremor, dysmetria, dysarthria, markedly hypotonia and weakness in all limbs. All deep tendon reflexes were diminished, flexor plantar reflexes were elicited. The cognition was relatively well preserved. He was attending main stream school with additional support. Cranial nerve examination revealed no abnormalities. Sensory system examination was normal apart from loss of join position sense in the inter-phalangeal joints in both lower limbs. We were unable to check for Romberg's sign. Examination of other systems was normal. Clinical diagnosis of AT was made.

Investigations: $\mathrm{Hb}-11.8 \mathrm{gm} \%$; Liver function testNormal; Total protein and albumin- normal; Calcium-10 gm\%; Chest X-ray- Normal.

Management: The diagnosis and prognosis were explained to the parents, and the child was commenced on regular multi-vitamin supplements and prophylactic dose of Penicillin- $\mathrm{V}$ to prevent sinopulmonary infections.

\section{Discussion}

AT, first described by Syllaba \& Henner ${ }^{4}$ and also known as Boder Sedgwick syndrome, is one of the most common inherited causes of childhood onset ataxia. An autosomal recessive neurodegenerative disease, its prevalence is reported to be 1 in 40,000-88,000 in the west ${ }^{5}$. The clinical phenotype is heterogeneous. The responsible 'ATM gene' has been mapped to chromosome 11q22-23. The ATM gene encodes a protein kinase which is the key regulator of cellular response to double strand breaks in DNA.

Ataxia and telangiectasia are the hallmarks of AT, and abnormal eye movements are universal findings. Early milestones are often normal until the child starts walking, when ataxia manifests. Symptoms include involuntary choreo-athetoid movements, oculomotor apraxia, drooling and dysarthria, frequent sino-pulmonary infections, increased incidence of malignancies (lymphomas and leukaemia) and diabetes mellitus ${ }^{3}$. The progressive neurodegeneration occurs from the progressive loss of purkinje cells in the cerebellum and the malfunctioning of other cells ${ }^{5}$. In most cases, the symptoms first appear before 5 years ${ }^{1}$. Late onset and milder variants are described where immunodeficiency, telangiectasia, sino-pulmonary infections and cancer may be absent or reduced, and slower progression of the neurological signs are seen ${ }^{3,6}$. Our patient is likely to belong to the late onset group.

Serum alpha fetoprotein (AFP) and carcinoembryonic antigen (CEA) are often elevated, Immunoglobulin A (IgA), IgE and IgG (G2 \& G4) levels are reduced in these children. Reduction of $\operatorname{IgA}$, the most prominent secretory immunoglobulin in the respiratory tract, may cause the frequent sinopulmonary infections. IgM level is normal or elevated ${ }^{3,7}$. Cell lines grown from these patients show increased radio-sensitivity, leading to chromosomal breakage and rearrangement ${ }^{8}$. This causes predisposition to risk of cancer in AT.

AT produces neurodegenerative changes mainly in the cerebellum. It causes significant morbidity, and death usually occurs from broncho-pulmonary infections or malignancy in early to late adolescence. Increased susceptibility in AT to ionising radiations must always be kept in mind while ordering radiological investigations.

\section{Differential diagnosis}

Friedreich's ataxia

Ataxia with oculomotor apraxia

Other autosomal recessive cerebellar ataxias ${ }^{1}$

Ataxic cerebral palsy.

\section{Evaluation of the management of this case in rural setting \& recommended management}

We relied on hallmark clinical signs to make the diagnosis. We were able to carry out basic investigations. Recommended investigations include genetic testing to confirm the diagnosis, immunological investigations, AFP, CEA, monitoring when malignancies are suspected, and neuro-imaging (MRI) in patients with unusual neurological course and/or when space occupying lesions are suspected. In resource-poor settings, many of these diagnostic tests are unavailable.

Currently there is no cure for AT. Patients require supportive therapy which consists of physiotherapy, occupational therapy, provision of orthoses, speech 
therapy, high calorie nutrition, gastrostomy when dysphagia ensues, prophylactic antibiotics and respiratory support when required ${ }^{3,7}$. Advice regarding high calorie nutrition, posture and physiotherapy as well as prophylactic antibiotics to prevent recurrent infections and vitamin supplementation was offered to our patient. There are limited options for rehabilitation therapy in rural settings such as ours.

Pharmacotherapy has been reported to be successful in individual cases, but there is no evidence to support their routine use. Gabapentine and clonazepam have been used to improve cerebellar tremors; Amantidine, Fluoxetine and Buspirone for motor incoordination and dysarthria; L-Dopa derivatives and Cholinergics, for managing chorea, dystonia and bradykinesia; and Baclofen, in dealing with spasticity and contractures ${ }^{9}$. IV immunoglobulins can be given if the level of IgA is low.

\section{Conclusion}

Careful clinical examination can help clinicians to diagnose rare neuro-degenerative conditions such as AT in rural settings. Appropriate medical treatment, regular physiotherapy and monitoring of the progress of such conditions, and ensuring adequate family and community support are the key components in managing these children in resource-poor rural settings.

\section{Author contribution}

Susan George wrote the first draft, and made the amendments. MC Mathews reviewed and corrected the initial drafts and the final paper.

Acknowledgement: We are grateful to the patient $\&$ his parents who gave consent for this publication and to all the staff at Dr. Megh Bahadur Parajuli Community
Hospital, Ilam as well as Himalayan Healthcare who support the paediatric care in Ilam.

\section{References}

1. Anheim M, Tranchant C, Koenig M. The autosomal recessive cerebellar ataxias. $N$ Engl J Med 2012;366(7):636-46.

2. Perlman S, Becker-Catania S, Gatti RA. Ataxiatelangiectasia: diagnosis and treatment. Semin Pediatr Neurol 2003;10(3):173-82.

3. Swaiman KF, Ashwal S, Ferriero DM, Schor NF. Swaiman's pediatric neurology: principles and practise. Fifth ed. Elsevier Saunders; 2012.

4. Syllaba L, Henner K. Contribution a l'independance de l'athetose double idiopathique et congenitale. Rev Neurol (Paris) 1926;1(541):562.

5. Lavin MF, Gueven N, Bottle S, Gatti RA. Current and potential therapeutic strategies for the treatment of ataxia-telangiectasia. Br Med Bull 2007;81$82: 129-47$.

6. Saviozzi S, Saluto A, Taylor AM, Last JI, Trebini F, Paradiso $M C$, et al. A late onset variant of ataxiatelangiectasia with a compound heterozygous genotype, A8030G/7481insA. J Med Genet 2002;39(1):57-61.

7. Kumar N, Aggarwal P, Dev N, Kumar G. Ataxia telangiectasia: learning from previous mistakes. BMJ Case Rep 2012;2012.

8. Lavin MF. Ataxia-telangiectasia: from a rare disorder to a paradigm for cell signalling and cancer. Nat Rev Mol Cell Biol 2008;9(10):759-69.

9. Hoche F, Seidel K, Theis M, Vlaho S, Schubert $\mathrm{R}$, Zielen $\mathrm{S}$, et al. Neurodegeneration in ataxia telangiectasia: what is new? What is evident? Neuropediatrics 2012;43(3):119-29. 